\title{
Self-imaging as an educational strategy
}

\section{Lia Zerbino, M. Lavagna, N. Baade}

Lia Maria Zerbino, M. E. Lavagna, N. N. Baade, "Self-imaging as an educational strategy," Proc. SPIE 3831, Sixth International Conference on Education and Training in Optics and Photonics, (16 June 2000); doi: $10.1117 / 12.388700$ 


\title{
Self-imaging like educational strategy
}

\author{
L. M. Zerbino ${ }^{\oplus \odot \odot}$, M. E. Lavagna ${ }^{\circledR \odot}$ and N. N. Baade ${ }^{\circledR \oplus}$ \\ OPTIMO \& IMApEC \\ Facultad de Ingeniería, Universidad Nacional de La Plata Argentina \\ E-mail: liaz@odin.ciop.unlp.edu.ar
}

Keywords: Self-imaging, conceptualization in optics, optic information concept

\begin{abstract}
In general, in the current programs of the Optics courses for the different teaching levels the diffraction and interference phenomena are included. The depth degree of the corresponding explanations depends on the mathematical knowledge of the students. Still in the basic university level, in our means, these tools don't allow the presentation of complete explanations based on the theory of the coherence and/or the scalar theory of the diffraction. However, as corollary of the treatment of those topics it is habitual, in the last years, the presentation of the subject of holography like application example and like a way to integrate the concepts of coherence, interference, diffraction and imaging formation, although this last aspect is only demonstrated by the practical experience. As an intermediate step, with the purpose of reinforcing the idea of the imaging formation for diffraction (Fourier images), it is interesting to show and to discuss self-imaging phenomena and some of their applications, before presenting the holography. The employment of this strategy in courses of professors' formation is commented.
\end{abstract}

\section{INTRODUCTION}

The constant concern of every educator is to answer the formational needs of generations to come. In our times, these demands are growing in importance day by day, since the evolution of scientific knowledge is bridging the temporal gap between what we call "science of today" and what will be "new science", therefore demanding a continuous update as regards not only contents but - more importantly - also concepts.

The adaptation of modern society to technological products that are now part of everyday life, demands that the educational system be organized so as to ensure that its products achieve, in a reasonable period of time, the level of conceptualization needed for them to feel dominant of that technology and not dependent from it. This intention should be evident from the very beginning of the educational process, so as to capture the interest of the learner. That is, educators are expected to find the way of guaranteeing or facilitating a learning that is the basis of a vision of the world open to advances and conceptual changes that will occur in the near future, and, above all, that compresses every educational stage of mankind.

The general concepts of synthesis, simulation, reconstruction, codification, recognition, and decodification are the basis of the technological developments that live with us today (every means of communication, information acquisition, transmission and processing, "virtual realities"). Nowadays our society is, in some aspects, "adapted" to these realities, in the sense that its members "know about" them, "know" them, and even "take advantage" of them. For example, many people is adapted to the sounds a synthesizer produces, they "know" that nowadays it is possible to simulate the sounds produced by the classical musical instruments of an orchestra, and that it is even possible to "invent" the sounds produced by a "virtual instrument"; some are "adapted" to holographic images, they "know" these are the image of something that never really "existed in our material space". However, the " " of the previous phrases can only be eliminated when the general concepts are significantly built, so that magic can leave place to thorough comprehension, which is the only basis of intellectual thinking and development. 
Nowadays it is not yet usual for large groups of the society to be familiar with optical information synthesis, perhaps because we are not yet surrounded by images of "non-existing objects". But in our university courses of General Physics it becomes evident that light has many characteristics which are similar to sound, and if the students who finish the course are not able to imagine or suppose that light synthesis might be carried out and taken advantage of, it is because they have not achieved a significant construction of those general concepts, among others.

\section{CONCEPTUALIZATION IN OPTICS}

The curricula of our General Physics courses includes the study of wave phenomena (elastic waves, electromagnetic waves, light and sound, interference, diffraction, polarization, Doppler effect). Traditional geometric optics are also studied, but without relation to the previous contents. Even in the cases where a "more modern" presentation is carried out, and the general mathematical treatment of the properties of waves of every kind, based on the superposition principle, is presented, Fourier analysis is shown only as a method to calculate the results of waves addition, an alternative to the algebraic method, to the complex method, and to the method of factors addition. However, polarization, interference and diffraction are analyzed and explained in a completely traditional way making use of these methods, without using or even mentioning Fourier analysis in the case the diffraction, be it Fraunhofer approximation or Fresnel approximation. In general, this reality is usually justified by making reference to the scarce mathematical tools that students at such early stages of their courses of studies are able to use. Even if we take that, since we are concerned mainly on the construction of the concepts rather than the calculation or development of demonstrations, we believe that one important consideration of this analysis is the one referring to experiments and applications commented upon during the course. As for the applications mentioned or the calculations and practical experiences proposed, these are more related to traditional interferometry and spectroscopy in the case of wave optics, and to the construction and design of traditional optical instruments in the case of Geometrical Optics. We even daresay that it is as if, apparently, their "update" reached the developments of the first quarter of the century. This description is reflected almost without changes in the basic bibliography of General Physics, and in the contents of pregrade plans used in our universities. In an attempt to bridge the gap, and in some cases as a way of incorporating a "modern" application of optics, by the end of the course practical demonstrations of holographic reconstruction are presented. This observation is accompanied by a graphical description of a simple experimental mounting (of the Leith - Upatnieks kind) for the construction of holograms and an explanation "with the hands" describing how interference and diffraction are combined to achieve that technique of registration and reconstruction of the wave front, commenting then on some applications. With no intention of diminishing the value of this attempt, that we have adopted ourselves, we have to acknowledge that, considering the results obtained, it is not enough to modify the conceptual background of students who, in general, only incorporate the knowledge that this technique exists, but whose justification remains in the field of magic. Consequently, they can hardly understand its applications.

How can they interpret, then, the advances already carried out and those which will come in the future, as regards optical processing of information, optical filtering, recognition of optical signals, and developments in active and adaptive optics, for example?

Which is the conceptual node of these advances that has apparently not been reached?

* Characteristics of the propagation of the luminous field

- Essential components of luminous information of an illuminated object.

- Techniques to detect and/or process optical information.

During the development of the course, elements that may contribute to this conceptual change are analyzed, but, perhaps, situations to confront them, or to force students to integrate these new concepts with other, pre-existent ones, have not been generated.

\section{TEACHING BY MEANS OF "PUZZLES"}

In an attempt to achieve this conceptual change with a constructivistic methodology, even setting aside the most complex mathematical developments, we have tried a strategy consisting in something like making the old concepts to "interfere" or "superpose", so as to produce in the mind of the students conceptual conflicts, puzzles, or paradoxical situations based on 
the imperfection or partiality of these previous concepts, and which will require the construction of the new concepts before students can solve, clarify or eliminate them.

The bases and steps of this methodology are:

* Presentation, formulation and recognition of situations that are enigmatic, surprising, conflictive or paradoxical if they are analyzed using the previous concepts.

* Deeper study of the puzzles based on the previous concepts. Detection of the conflict.

- Formulation of the new theories and models to solve the puzzle.

- Elucidation of the puzzle.

- Prediction of pseudo-puzzles based on the same conflicts, now overcame (they would be puzzles if the new conceptualization had not been reached)

Undoubtedly, the presentation of holography, for example, awakens the interest and curiosity of students, and this can be exploited to achieve a conceptual change, always by directing them to relate the already acquired concepts, and without limiting them by saying that the explanation of these new concepts is too complicated for their level of knowledge development. The presentation of holography is, on the light of previous knowledge students have, a shooting "puzzle" that may cause this integration of previous concepts to build up the new ones.

The elements of the puzzle, from the questions or doubts students have, are of the type: Where is the object? Where does the image "come from"? What is there in the holographic plate? How can I be seeing an image if there is no object and no lens? Can an interference diagram produce an image? What has diffraction have to do with image formation? Why do I see what I see? Why do I see three-dimensional objects?, etc. Interference, diffraction and image formation are apparently in conflict, but the fact is that there is a dark conceptual area: What is an image? What is it what we call image? How is it formed? Questions that are not even posed, but that are underlying in the core of the conflict.

We have found that the production and analysis of a previous "puzzle", showing Talbot sel-images, is extremely useful for this strategy.

\section{EXPERIMENTS AND RESULTS}

To test this strategy we have carried out the experiment during a workshop with the participation of young teachers and assistant teachers whose knowledge of optics was limited to those pointed out above. They consented, as a preliminary step, to "play students" to be able to discuss later the advantages and possibilities for the presentation of this strategy in ordinary courses.

The comments gathering the results obtained are now summarized.

When observing the simple experiment of the formation of Talbot self-images with Ronchi rulings, conflict and surprise are evident. Doubts are of the type: Why are there images? What is making them, since there are no lenses? If an illuminated grating produces a diffraction pattern, why do I see images of the grating?, etc. And this time: What is an image? When do I say that something is an image? Or How do I know that something is an image?... and the process of relating concepts and knowledge begins: is the image "point by point"? What makes it possible to recognize the grating? Which is the information that makes me recognize the grating? What is there in the intermediate planes of what we call images? Which is the spatial frequency of the grating? What are the information contents of the different planes?, etc.

Even without a mathematical treatment or a rigorous description, this phenomenon can be analyzed in terms of the spatial frequency components of the object grating, the propagation of the diffracted field, and the composition of spatial frequencies.

This discussion allows the conceptualization of the decomposition and synthesis of spatial frequencies, the recomposition of the emerging wave front of the object grating, which is visualized as a replication or image despite the fact that it is not point by point.

At the same time, this translates the puzzle to questioning what is it that makes the lens focus and then discuss Abbe's theory of image formation. Thus, a wide range of ideas that may be verified with simple experiments arises: recognition of the spatial frequency components of an object in the focal plane of a lens, filtering of spatial frequencies, recognition of information about the object outside the plane of a "perfect image", Talbot interferometry, temporal and spatial coherence, Lau self-images, etc. All of which would be also puzzles for any subject not going first through this "path". These are now what we previously called "pseudo-puzzles". 
Thus, with the answers and concepts discussed, the concept of image gradually loses its "importance" to leave room for the notion of optical information.

After the discussion, when introducing the holographic demonstration, the conceptual "jump" required to integrate it to previous conceptions is not as huge.

The experiment carried out with a group of teachers demonstrated that even though they had no previous knowledge as regards holography or of the special characteristics and orders diffracted by a sinusoidal grating, they rapidly center their attention on the problem of justifying the reconstruction of the wave front of the object from the optical information "kept", "contained", or "registered" in the holographic plate in such a way that its propagation would yield the effective reconstruction of the wave front they saw.

\section{CONCLUSIONS}

With this preliminary experiment we have found that the reactions and comments of the participants to whom the experiments were presented in the order here porposed, allow us to conclude that:

* the inclusion of the consideration of the self-images phenomenon caused a conceptual change and an integration of concepts they believed were not connected.

* holography can be then introduced, and the previous conceptual changes can be used to take students one step forward.

* these conceptual advances allowed the group to strengthen their confidence in the possibility of "reasoning" over the foundations of some current technological applications in the field of optics, and their future.

\section{ACKNOWLEDGEMENTS}

The authors would like to thank all the teachers who participated in the preliminary workshop and to the teachers who participated in the didactic experiment.

\section{BIBLIOGRAPHY}

- K Patorski. The self-imaging phenomenon and its applications. Progress in Optics XXVII, Ed. E. Wolf. North Holland, Amsterdam 1989.

- $\quad \boldsymbol{R}$. F. Edgar. The Fresnel diffraction image of periodic structures. Optica Acta . 16, 281(1969).

- J. T. Winthrop and C. R. Worthington. Theory of Fresnel images. I. Plane periodic objects in monochromatic light. $J$. Opt. Soc. Am. 55, 373-381 (1965).

- M. Born and E. Wolf. Principles of optics. Pergamon Press. 1965.

- A. W. Lohmann. A new Fourier spectrometer consisting of two-gratings-interferometer. K. J. Habbell Ed., London, 1961 Butterworth.

- A. W. Lohmann, J. Ojeda-Castañeda and E. E. Sicre. Multiple interaction bandstop filters based on the Talbot effect. Opt. Commun.49, 388 (1984).

- J. Ibarra and J. Ojeda-Castañeda. Talbot interferometry: a new geometry. Opt. Commun.96, 294 (1993)

- D. E. Silva. A simple interferometric method of beam collimation. Appl. Opt., 10, 980 (1971).

(1) IMAPEC, Facultad de Ingeniería Universidad Nacional de La Plata, Argentina

(2) OPTIMO, Facultad de Ingeniería Universidad Nacional de La Plata, Argentina

(3) Facultad Regional La Plata Universidad Tecnológica Nacional (UTN), Argentina

(4) Centro de Investigaciones Opticas (CIOp), Argentina

(5) Consejo Nacional de Investigaciones Científicas y Técnicas. (CONICET), Argentina 